\title{
OPTICAL OBSERVATIONS OF NEARBY INTERSTELLAR GAS
}

\author{
P.C. Frisch and D.G. York \\ Astronomy and Astrophysics Center, University of Chicago
}

The yellow $D$ lines from neutral sodium are some of the most useful interstellar absorption features for tracing out low density optically thin interstellar material. Hobbs (1978) compared column densities of $\mathrm{NaI}$ as determined from the $\mathrm{D}$ lines with $\mathrm{HI}$ column densities derived from ultraviolet observations and found values of $N(\mathrm{NaI}) / \mathrm{N}(\mathrm{H})<6.3 \times 10^{-9}$ in 18 sight-lines with very low reddening, with the exact value of the ratio apparently a function of temperature. If this ratio limit holds for diffuse clouds in nearby space, we expect that observations of the $\mathrm{NaI} \mathrm{D}_{2}$ lines at the $3 \mathrm{~mA}$ level can be used to trace ${ }_{2}$ out nearby clouds with column densities in the range of $\geq 2.5 \times 10^{10} \mathrm{~cm}^{-2}$. Thus sensitive measurements of the NaI D lines are a useful way of observing nearby neutral cloud material. With this in mind, we have chosen high-resolution observations of the yellow sodium lines as a means of locating and mapping nearby low density interstellar clouds.

In the past, most interstellar line observations have relied on 0 and $B$ stars as target objects. Since these stars generally fall in associations that lie outside of the nearest 100 parsecs, there is no a priori way of identifying those interstellar absorption features which are formed in ciouds within the nearest 100 parsecs. In order to determine the distance of nearby clouds, we have singled out relatively abundant nearby cool stars as target objects. The distance of the interstellar cloud is then determined by observing a sequence of stars spaced in distance along one line of sight. The distance of the nearest stars in which the interstellar absorption feature is seen provides an estimate of the distance of the cloud. Most of the distances we have used so far are based on the spectra of the target stars, and are therefore not known accurately. However, the upcoming astrometric Hipparcos satellite combined with the next generation of ground-based astrometric telescopes should allow the determination of stellar distances out to 100 parsecs more accurately.

The results of applying this technique to the clouds in the sight-line towards $\zeta$ Oph are shown in Figure 1 . This star is located 170 parsecs from the Sun with probably the most well-studied interstellar spectrum of any star. We searched for, and found, surprisingly strong interstellar $\mathrm{NaI} \mathrm{D}_{1}$ and $\mathrm{D}_{2}$ absorption lines in three stars within two degrees or less from the sight line to $\zeta_{5} 0 \mathrm{ph}$, and with distances less than 100 parsecs from the Sun. A fourth star with a slightly greater separation from the 5 Oph sight-line, but within 56 parsecs of the Sun, produced no detection of interstellar material. These $\mathrm{NaI} \mathrm{D}_{1}$ absorption features are shown in Fig. 1 where intensity is plotted versus hel iocentric velocity. These data were taken using the McDonald Observatory coude Digicon detector at a resolution of $3 \mathrm{~km} / \mathrm{s}$. Information about the stars are given in the Table. The stellar distances listed in the Table are all spectral 
distances and taken from Sky Catalogue 2000.0. Interstellar absorption features are seen in al but the nearest of these stars, 20 oph. The broad absorption feature seen in that star is stellar. Broad, very shallow, stellar NaI absorption features are also found for the stars HD149662 and HD149807 on tracings of a larger velocity interval of the spectral continua. Also note that the weak absorption features seen between +15 and $+40 \mathrm{~km} / \mathrm{s}$ in all of the stars are telluric features from the earth's atmosphere.

In the $2 \times 3$ degree region of the sky defined by the four stars showing $\mathrm{NaI}$ absorption, two distinct cloud complexes are present centered near $-14 \mathrm{~km} / \mathrm{s}$ and $-28 \mathrm{~km} / \mathrm{s}$ (heliocentric velocity). Ultraviolet observations of $\zeta$ Oph show that each of these two NaI features is actually made up of several individual velocity features with distinct ionization properties (Morton 1975). Maps of 21-cm emission at $0 \mathrm{~km} / \mathrm{s}$ and $-14 \mathrm{~km} / \mathrm{s}$ in the local standard of rest (corresponding to the two $\mathrm{NaI}$ clouds) in this $2 \times 3$ degree region do not show pronounced patchiness that could explain the variations in the line profiles seen in the Figure. (Cleary et. al. 1979) Based on the fact that one star, HD149108, shows only the $-28 \mathrm{~km} / \mathrm{s}$ cloud, while the other stars show both clouds, we thus conclude that the spectral star distances Iisted in the Table may not be correct, since the interstellar line strengths should increase in strength with star distance. The relative star positions implied by the interstellar lines, starting with the nearest stars, are 20 Oph, HD149108, HD149807, HD149662 and $\zeta$ Oph.

This narrow unsaturated $\mathrm{Na}_{1}$ feature in HD149108 has a column density of $\mathrm{N}(\mathrm{NaI}) \sim 3 \times 10_{19}^{11} \mathrm{~cm}_{2}^{-2}$, corresponding to a hydrogren column density of $\mathrm{N}(\mathrm{HI}) \geq 5 \times 10^{19} \mathrm{~cm}^{-2}$, providing the ratio limit quoted above from Hobbs (1978) encompasses the value for this cloud. According to the stellar distances entered in the Table, this cloud is certainly within 80 parsecs of the Sun, and within 55 parsecs if the distance to HD149662 is correct, in agreement with previous conclusions that this $-28 \mathrm{~km} / \mathrm{s}$ feature is near the Sun (Frisch 1981, Crutcher 1982).

Another point to note from these data is that material associated with some portion of the main interstellar absorption feature at $\sim-14 \mathrm{~km} / \mathrm{s}$ found in $\zeta$ oph must also lie within the nearest 100 parsecs. This strong feature has substantial amounts of $\mathrm{H}_{2}$ as well as other interstellar molecules associated with it and is the prototypical diffuse cloud (Herbig 1968, Morton 1975). Based on the two stars H0149807 and HD149108, at estimated distances of about 80 parsecs and which both show distinct absorption from this -14 $\mathrm{km} / \mathrm{s}$ gas, we conclude that this main feature is actually a cloud complex and that part(s) of this complex are within 100 parsecs.

A third thing to note from these data is that the variation in the strength of the $-28 \mathrm{~km} / \mathrm{s}$ cloud between the different stars indicates some patchiness is present within diffuse clouds on scales of the order of a parsec or less. 
In summary, these observations indicate that a cloud with a heliocentric velocity of $\sim-28 \mathrm{~km} / \mathrm{s}$ and a hydrogen column density that possibly could be on the order of, or greater than, $5 \times 10^{29}$ $\mathrm{cm}^{-2}$ is located within the nearest 50-80 parsecs in the direction of Ophiuchus. This is a surprisingly large column density of material for this distance range. The patchy nature of the absorption from the cloud indicates that it may not be a feature with uniform properties, but rather one with small scale structure which includes local enhancements in the column density. This cloud is probably associated with the interstellar cloud at about the same velocity in front of the 20 parsec distant star a Oph (Frisch 1981, Crutcher 1982), and the weak interstellar polarization found in stars as near as 35 parsecs in this general region (Tinbergen 1982). These data also indicate that some portion of the $-14 \mathrm{~km} / \mathrm{s}$ cloud also must lie within the 100 parsec region.

Similar observations of both $\mathrm{NaI}$ and CaII interstellar absorption features have been performed in other lines of sight. We find similar interstellar absorption features in a dozen stars between 20 and 100 parsecs of the Sun, and located in Quadrants IV, I and II of the galaxy. A number of these stars also show surprisingly strong $\mathrm{NaI}$ absorption lines in light of their reported distances.

\section{References}

Cleary, M.N., Heiles, C., and Haslam, C.Ǵ.T. 1979, Astr. Ap. Sup., $36,95$.

Crutcher, R.M. 1982, Ap. J. 254, 82.

Frisch, P.C. 1981, Nature, 293, 377.

Herbig, G.H. 1968, Zs. F. Ap., 68, 243.

Hirshfeld, A., and Sinnott, R.W.(ed.) 1982, Sky Catalogue 2000.0

(Cambridge University Press and Sky Publishing Corporation)

Hobbs, L.M. 1978, Ap. J., 222, 491.

Morton, D.C. 1975, Ap. J., 197, 85.

Tinbergen, J. 1982, Astro. Ap., 105, 53. 
Table: Stellar Data

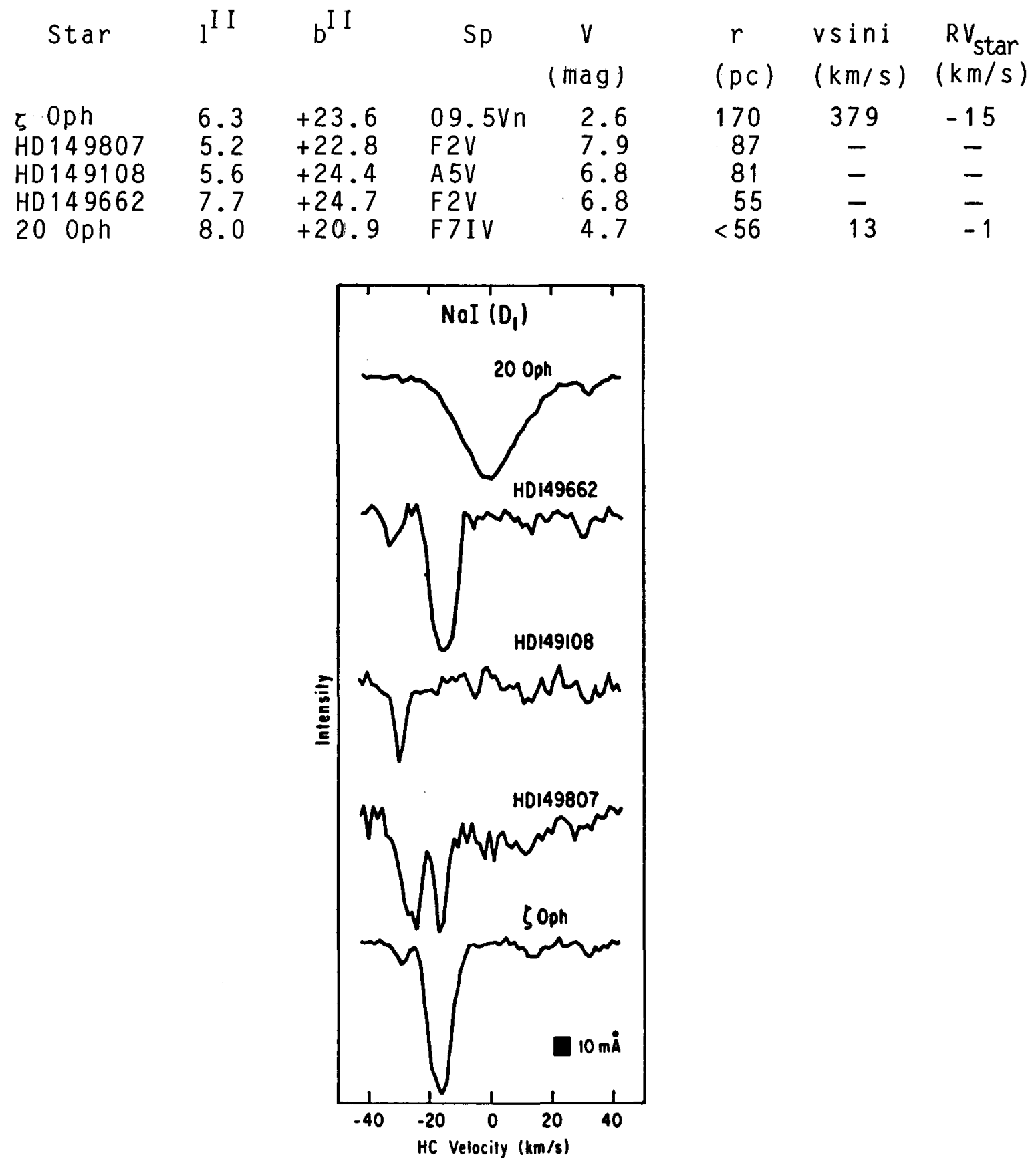

